\section{TATRA \\ MOUNTaiNS \\ Mathematical Publications}

DOI: $10.2478 /$ tmmp-2013-0033

Tatra Mt. Math. Publ. 57 (2013), 21-33

\title{
LOGARITHMIC SIGNATURES FOR ABELIAN GROUPS AND THEIR FACTORIZATION
}

\author{
Pavol Svaba - Tran van Trung - Paul Wolf
}

\begin{abstract}
Factorizable logarithmic signatures for finite groups are the essential component of the cryptosystems $\mathrm{MST}_{1}$ and $\mathrm{MST}_{3}$. The problem of finding efficient algorithms for factoring group elements with respect to a given class of logarithmic signatures is therefore of vital importance in the investigation of these cryptosystems. In this paper we are concerned about the factorization algorithms with respect to transversal and fused transversal logarithmic signatures for finite abelian groups. More precisely we present algorithms and their complexity for factoring group elements with respect to these classes of logarithmic signatures. In particular, we show a factoring algorithm with respect to the class of fused transversal logarithmic signatures and also its complexity based on an idea of Blackburn, Cid and Mullan for finite abelian groups.
\end{abstract}

\section{Introduction}

Logarithmic signatures and covers for finite groups have found interesting applications in designing cryptographic primitives and pseudo-random number generators [2]-7], 9], [11. Logarithmic signatures and covers are a kind of factorization of a finite group $G$ through its subsets and they induce surjective mappings from $\mathbb{Z}_{|\mathrm{G}|}$ onto $G$. An interesting fact is that these mappings can, in general, be computed very efficiently. However, if we take a random cover for a finite group, its induced mapping behaves like a random function, see [9], thus inverting this mapping becomes an intractable problem. There are strong indications supporting this fact. On the other hand, the mapping induced by a logarithmic signature actually is a bijection. As there are various classes of logarithmic signatures which have arisen from algebraic structures of groups, the problem of inverting this bijection needs a careful study. More important is the fact that logarithmic signatures, whose induced mappings are used as part of the private key in a public key cryptosystem have to be efficiently invertible,

(C) 2013 Mathematical Institute, Slovak Academy of Sciences.

2010 Mathematics Subject Classification: 94A60, $20 \mathrm{~K} 01$.

Keywords: factorization, fused transversal logarithmic signature, finite group. 
see [2], 7], [1]. Hence, the question of inverting the induced bijection for a given logarithmic signature is of significance. In [6] M a g liver a s and M e m o n have shown that the induced bijections for a specific class of transversal logarithmic signatures derived from a chain of point stabilizer subgroups for permutation groups of degree $n$ are invertible with a time complexity of $O\left(n^{2}\right)$. In [8] it is shown that the induced bijection of a certain specific class of transversal logarithmic signatures for elementary abelian 2-groups can be inverted with a time complexity of $\mathrm{O}(1)$, see also [13. In [1] B l a c k b u r n, $\mathrm{C}$ i d and $\mathrm{M} \mathrm{ull} \mathrm{an} \mathrm{intro-}$ duce a method for inverting induced bijections of fused transversal logarithmic signatures for elementary abelian 2-groups. In [11] the problem of inverting these induced bijections is also discussed.

In this paper we study the inverting problem of the bijections induced from transversal and fused transversal logarithmic signatures for abelian groups. We present algorithms and their complexity for the inverting problem. In particular, we show an algorithm based on the idea of B la c k b u r n et al. and determine its complexity. We further study the inverting problem by using trapdoor information and show that fused transversal logarithmic signatures for abelian groups are tame with respect to this method.

\section{Preliminaries}

In this section we briefly present notation, definitions and some basic facts about logarithmic signatures and covers for finite groups and their induced mappings. For more details the reader is referred to [6], [7]. We assume that the reader is familiar with the basics of group theory. The group theoretic notation used is standard and may be found in any textbook of group theory. In this paper we only deal with finite groups.

Let $\mathrm{G}$ be a finite group. We define the width of $\mathrm{G}$ to be the positive integer $w=\left\lceil\log _{2}|G|\right\rceil$. Suppose that $\alpha=\left[A_{1}, A_{2}, \ldots, A_{s}\right]$ is a sequence of subsets $A_{i}=\left[a_{i 1}, \ldots, a_{i r_{i}}\right] \subset G$, such that $\sum_{i=1}^{s}\left|A_{i}\right|$ is polynomially bounded in the width $w$ of $\mathrm{G}$. Let $\mathcal{S}$ be a subset of $\mathrm{G}$. We say that $\alpha$ is a cover for $\mathcal{S}$ if every product $a_{1 j_{1}} \ldots a_{s j_{s}}$ lies in $\mathcal{S}$ and if each element $g \in \mathcal{S}$ can be expressed in at least one way as a product of the form

with $a_{i j_{i}} \in A_{i}$.

$$
g=a_{1 j_{1}} \ldots a_{s j_{s}}
$$

If every $g \in \mathcal{S}$ can be expressed in exactly one way by the equation (2.1), then $\alpha$ is called a logarithmic signature (LS) for $\mathcal{S}$. If $\mathcal{S}=\mathrm{G}, \alpha$ is called a cover, resp., a logarithmic signature for $\mathrm{G}$.

The $A_{i}$ are called the blocks, and the vector $\left(r_{1}, \ldots, r_{s}\right)$ with $r_{i}=\left|A_{i}\right|$ the type of $\alpha$. We say that $\alpha$ is nontrivial if $s \geqslant 2$ and $r_{i} \geqslant 2$ for $1 \leqslant i \leqslant s$; otherwise $\alpha$ is said to be trivial. The $\operatorname{sum} \ell(\alpha)=\sum_{i=1}^{s} r_{i}$ is defined as the length of $\alpha$. 


\section{LOGARITHMIC SIGNATURES FOR ABELIAN GROUPS...}

Let $\Gamma=\left\{\left(\mathcal{G}_{\ell}, \alpha_{\ell}\right)\right\}_{\ell \in \mathbb{N}}$ be a family of pairs, indexed by the security parameter $\ell$, where the $\mathcal{G}_{\ell}$ are groups in a common representation, and where $\alpha_{\ell}$ is a specific cover for $\mathcal{G}_{\ell}$ of length polynomial in $\ell$. We say that $\Gamma$ is tame if there exists a probabilistic polynomial time algorithm $\mathcal{A}$ such that for each $g \in \mathcal{G}_{\ell}, \mathcal{A}$ accepts $\left(\alpha_{\ell}, g\right)$ as input, and outputs a factorization $\varphi(g)$ of $g$ with respect to $\alpha_{\ell}$ (as in equation (2.1) with overwhelming probability of success. We say that $\Gamma$ is wild if for any probabilistic polynomial time algorithm $\mathcal{A}$, the probability that $\mathcal{A}$ succeeds in factorizing a random element $\mathrm{g}$ of $\mathrm{G}$ is negligible.

Let $\gamma: \mathrm{G}=\mathrm{G}_{0}>\mathrm{G}_{1}>\cdots>\mathrm{G}_{\mathrm{s}}=1$ be a chain of subgroups of $\mathrm{G}$, and let $A_{i}$ be an ordered, complete set of right (or left) coset representatives of $G_{i}$ in $G_{i-1}$. Then it is clear that $\left[A_{1}, \ldots, A_{s}\right]$ forms a logarithmic signature for $G$, called a transversal logarithmic signature (TLS).

Let $\mathrm{G}$ be a permutation group on the set $X=\{1, \ldots, n\}$. Consider a chain of nested point stabilizers $\mathrm{G}=\mathrm{G}_{0}>\mathrm{G}_{1}>\cdots>\mathrm{G}_{\mathrm{s}}=1$, where $\mathrm{G}_{\mathrm{i}}$ fixes pointwise the symbols $1,2, \ldots, i$, for any $i \geqslant 1$. It is shown in 6 that a specific constructed class of transversal logarithmic signatures from this chain of subgroups has a factorization with a time complexity of $\mathrm{O}\left(\mathrm{n}^{2}\right)$. In general, the problem of finding a factorization in equation (2.1) with respect to a given cover is presumedly intractable. There is strong evidence in support of the hardness of the problem. For example, let $\mathrm{G}$ be a cyclic group and $\mathrm{g}$ be a generator of $\mathrm{G}$. Let $\alpha=\left[A_{1}, A_{2}, \ldots, A_{s}\right]$ be any cover for $G$, for which the elements of $A_{i}$ are written as powers of $\mathrm{g}$. Then the factorization with respect to $\alpha$ amounts to solving the Discrete Logarithm Problem in G.

The main point making covers and LS interesting for use in cryptography is that if the above factorization problem is intractable, they essentially induce one-way functions. This can be described as follows. Let $\alpha=\left[A_{1}, A_{2}, \ldots, A_{s}\right]$ be a cover of type $\left(r_{1}, r_{2}, \ldots, r_{s}\right)$ for $G$ with $A_{i}=\left[a_{i, 1}, a_{i, 2}, \ldots, a_{i, r_{i}}\right]$ and let $m=\prod_{i=1}^{s} r_{i}$. Let $m_{1}=1$ and $m_{i}=\prod_{j=1}^{i-1} r_{j}$ for $i=2, \ldots, s$. Let $\tau$ denote the canonical bijection from $\mathbb{Z}_{\mathrm{r}_{1}} \oplus \mathbb{Z}_{\mathrm{r}_{2}} \oplus \cdots \oplus \mathbb{Z}_{\mathrm{r}_{\mathrm{s}}}$ on $\mathbb{Z}_{\mathrm{m}}$; i.e.,

$$
\begin{gathered}
\tau: \mathbb{Z}_{\mathrm{r}_{1}} \oplus \mathbb{Z}_{\mathrm{r}_{2}} \oplus \cdots \oplus \mathbb{Z}_{\mathrm{r}_{\mathrm{s}}} \rightarrow \mathbb{Z}_{\mathrm{m}}, \\
\tau\left(j_{1}, j_{2}, \ldots, j_{\mathrm{s}}\right):=\sum_{i=1}^{s} j_{i} \mathrm{~m}_{\mathrm{i}} .
\end{gathered}
$$

Using $\tau$ we now define the surjective mapping $\breve{\alpha}$ induced by $\alpha$.

$$
\begin{gathered}
\breve{\alpha}: \mathbb{Z}_{\mathrm{m}} \rightarrow \mathrm{G}, \\
\breve{\alpha}(x):=\mathrm{a}_{1, j_{1}} \cdot \mathrm{a}_{2, j_{2}} \cdots \mathrm{a}_{\mathrm{s}, \mathrm{j}_{\mathrm{s}}},
\end{gathered}
$$

where $\left(j_{1}, j_{2}, \ldots, j_{s}\right)=\tau^{-1}(x)$. Since $\tau$ and $\tau^{-1}$ are efficiently computable, the mapping $\breve{\alpha}(x)$ is efficiently computable. 


\section{PAVOL SVABA - TRAN VAN TRUNG - PAUL WOLF}

Conversely, given a cover $\alpha$ and an element $y \in G$, to determine any element $x \in \breve{\alpha}^{-1}(y)$ it is necessary to obtain any one of the possible factorizations of the type (2.1) for $y$ and determine indices $j_{1}, j_{2}, \ldots, j_{s}$ such that $y=a_{1, j_{1}} \cdot a_{2, j_{2}} \cdots a_{s, j_{s}}$. This is possible if and only if $\alpha$ is factorizable. Once a vector $\left(\mathbf{j}_{1}, \mathfrak{j}_{2}, \ldots, \mathfrak{j}_{s}\right)$ has been determined, $\breve{\alpha}^{-1}(y)=\tau\left(\mathbf{j}_{1}, \mathfrak{j}_{2}, \ldots, \mathbf{j}_{s}\right)$ can be computed efficiently.

Assume that $\alpha=\left[A_{1}, A_{2}, \ldots, A_{s}\right]$ is a cover for $G$. Let $g_{0}, g_{1}, \ldots, g_{s} \in G$, and consider $\beta=\left[B_{1}, B_{2}, \ldots, B_{s}\right]$ with $B_{i}=g_{i-1}^{-1} A_{i} g_{i}$. We say that $\beta$ is a two sided transform of $\alpha$ by $g_{0}, g_{1}, \ldots, g_{s}$; in the special case, where $g_{0}=1$ and $g_{s}=1$, $\beta$ is called a sandwich of $\alpha$. It is clear that $\beta$ is a cover for $\mathcal{G}$.

Two covers (logarithmic signatures) $\alpha, \beta$ are said to be equivalent if $\breve{\alpha}=\breve{\beta}$. For example, if $\beta$ is a sandwich of $\alpha$, then $\alpha$ and $\beta$ are obviously equivalent.

A block $A_{i}$ of a cover is called normalized if $A_{i}$ contains the identity element of the group, i.e., $i d_{G} \in A_{i}$. It is obvious that by using a sandwich transformation with $g_{i} \in A_{i}$ for $i=1, \ldots, s-1$ we can transform $\alpha$ to an equivalent $\beta$ having all $(s-1)$ blocks normalized, the last block $B_{s}$ is in general not normalized.

Let $\alpha=\left[A_{1}, A_{2}, \ldots, A_{s}\right]$ be a LS for a finite group $G$. Consider $k$ blocks $A_{i_{1}}, \ldots, A_{i_{k}}$ of $\alpha$. Define

$$
B:=A_{i_{1}} \cdot A_{i_{2}} \cdots A_{i_{k}}=\left\{a_{i_{1}} \cdot a_{i_{2}} \cdots a_{i_{k}} \mid a_{i_{j}} \in A_{i_{j}}, j=1, \ldots, k\right\} .
$$

We call $B$ a fused block of $A_{i_{1}}, \ldots, A_{i_{k}}$. If we apply fusion operations to the blocks of $\alpha$ we generally obtain a cover $\beta=\left[B_{1}, \ldots, B_{t}\right]$ for a subset of $G$, where $t<s$. However, if $G$ is abelian, then $\beta$ remains a LS for $G$. Usually $\beta$ may not necessarily be equivalent to $\alpha$, and we call $\beta$ a fused logarithmic signature of $\alpha$.

In the rest of the paper we assume that multiplication in the groups is taken to be constant.

\section{Algorithms for factorization with respect to TLS}

In this section we present algorithms for factorization with respect to TLS for finite groups.

We first present a generic algorithm for factoring with respect to any TLS $\alpha$ for any group G (abelian or non-abelian).

The number of steps required for the algorithm is $O\left(\sum_{i=1}^{s}\left|A_{i}\right|\right)$. If $G$ is a permutation group of degree $n$, there exist algorithms for solving the membership problem for $\mathrm{G}$ in polynomial time with respect to $\mathrm{n}$ by using a strong generating set.

Now let $G$ be a finite abelian group. In the following we show that there is a factoring algorithm for TLS of G having a time complexity of $\mathrm{O}(w)$. 
Algorithm 1 Generic Algorithm

Input: $\mathcal{G}$ : a finite group, $\alpha=\left[A_{1}, A_{2}, \ldots, A_{s}\right]$ a TLS for $G$ constructed from a chain of subgroups $\mathrm{G}=\mathrm{G}_{0}>\mathrm{G}_{1}>\cdots>\mathrm{G}_{\mathrm{s}}=1$ of $\mathrm{G}, \mathrm{g} \in \mathrm{G}$.

Output: $a_{i} \in A_{i}$ such that $g=a_{1} \ldots a_{s}$.

1: Find a unique element $a_{1} \in A_{1}$ such that $g_{1}=a_{1} \cdot g^{-1} \in G_{2}$. Find a unique element $a_{2} \in A_{2}$ such that $g_{2}=a_{2} \cdot g_{1}^{-1} \in G_{3}$. Continue this process until $A_{s}$. Then we have $g=a_{1} \ldots a_{s}$ as a factorization of $g$ with respect to $\alpha$.

Again let $\alpha=\left[A_{1}, A_{2}, \ldots, A_{s}\right]$ be a TLS for $G$ constructed from a chain of subgroups $G=G_{0}>G_{1}>\cdots>G_{s}=1$. Since $G$ is abelian, each $G_{i}$ is a normal subgroup of $G$. Therefore we can form the quotient group $\bar{G}^{(i)}:=G / G_{i}$ for $i=0, \ldots, s$, where

$$
\overline{\mathrm{G}}^{(\mathrm{i})}:=\mathrm{G} / \mathrm{G}_{\mathrm{i}}=\left\{\mathrm{G}_{\mathrm{i}} \cdot \mathrm{g} \mid \mathrm{g} \in \mathrm{G}\right\} .
$$

The elements of $\overline{\mathrm{G}}^{(\mathfrak{i})}$ are denoted by $\overline{\mathrm{g}}^{(\mathfrak{i})}$, where

$$
\overline{\mathrm{g}}^{(\mathrm{i})}=\phi^{(\mathrm{i})}(\mathrm{g}) \text { and } \phi^{(\mathrm{i})}: \mathrm{G} \longrightarrow \overline{\mathrm{G}}^{(\mathrm{i})}
$$

defined by $\phi^{(i)}(g)=G_{i} \cdot g$ is the canonical homomorphism.

For each $i=1, \ldots, s$ define $\bar{\alpha}^{(i)}=\left[\bar{A}_{1}^{(i)}, \ldots, \bar{A}_{i}^{(i)}\right]$ with $\bar{A}_{j}^{(i)}=\phi^{(i)}\left(A_{j}\right)$. Note that the blocks $\bar{A}_{i+1}^{(i)}, \ldots, \bar{A}_{s}^{(i)}$ in the quotient group $\bar{G}^{(i)}$ are viewed as blocks of size 1 with the identity as their unique element. Therefore we ignore them all. For each $i=1, \ldots, s$ define $\pi_{i}$ to be the permutation in $S_{r_{i}}$ which sorts the elements of $A_{i}$ according to a certain order, for instance, numerical order. When applying $\pi_{i}$ to $A_{i}$ for all $i=1, \ldots, s$ we obtain a TLS $\beta=\left[B_{1}, B_{2}, \ldots, B_{s}\right]$. The factorization with respect to $\alpha$ can obviously be done via $\beta$ and $\pi_{i}$. Precisely, if $\mathrm{g}=\mathrm{a}_{1 j_{1}} \ldots \mathrm{a}_{\mathrm{s} j_{\mathrm{s}}}$ is a factorization of an element $\mathrm{g} \in \mathrm{G}$ with respect to $\beta$, then $g=a_{1 \pi_{1}^{-1}\left(j_{1}\right)} \ldots a_{s \pi_{s}^{-1}\left(j_{s}\right)}$ is a factorization with respect to $\alpha$, where $\pi_{i}^{-1}$ is the inverse of $\pi_{i}$. We now present an algorithm for factoring with respect to a sorted TLS.

The main complexity of the factorization in the step $i$ depends on the search of element $\bar{a}_{i}^{(i)}$ in $\bar{A}_{i}^{(i)}$. This can be done in time of $O\left(\log _{2}\left|A_{i}\right|\right)$, since the elements of $A_{i}$ are sorted. Hence

$$
\mathrm{O}\left(\sum_{i=1}^{s} \log _{2}\left|A_{i}\right|\right)=\mathrm{O}(w)
$$

is the complexity of Algorithm 2. The only extra operation for factoring with respect to an unsorted TLS is the application of the inverse permutations $\pi_{i}^{-1}$ to the result obtained from a sorted TLS, as discussed above. Moreover, computing with each $\pi_{i}$ can be carried out in constant time. Hence, we obtain the following theorem as a consequence of Algorithm 2. 


\section{Algorithm 2 Factorization with TLS}

Input: $G$ : abelian group, $\alpha=\left[A_{1}, A_{2}, \ldots, A_{s}\right]$ a sorted TLS for $G$ constructed from a chain of subgroups $\mathrm{G}=\mathrm{G}_{0}>\mathrm{G}_{1}>\cdots>\mathrm{G}_{\mathrm{s}}=1$ of $\mathrm{G}, \mathrm{g} \in \mathrm{G}$.

Output: $a_{i} \in A_{i}$ such that $g=a_{1} \ldots a_{s}$.

1: Using the chain of quotient groups $\overline{\mathrm{G}}^{(s-1)}, \ldots, \overline{\mathrm{G}}^{(1)}$, the chain of TLS $\bar{\alpha}^{(s-1)}, \ldots, \bar{\alpha}^{(1)}$, and the chain of elements $\bar{g}^{(s-1)}, \ldots, \bar{g}^{(1)}$, we carry out the factorization of $\mathrm{g}$ as follows.

First, find the unique element $\overline{\mathrm{a}}_{1}^{(1)} \in \bar{\alpha}^{(1)}=\left[\overline{\mathrm{A}}_{1}^{(1)}\right]$ such that $\overline{\mathrm{g}}^{(1)}=\overline{\mathrm{a}}_{1}^{(1)}$ (note that $\bar{A}_{1}^{(1)}$ is identical to the quotient group $\mathrm{G} / \mathrm{G}_{1}:=\overline{\mathrm{G}}^{(1)}$ ).

In the quotient group $\overline{\mathrm{G}}^{(2)}$ we have $\bar{\alpha}^{(2)}=\left[\overline{\mathrm{A}}_{1}^{(2)}, \overline{\mathrm{A}}_{2}^{(2)}\right]$ and the element $\overline{\mathrm{g}}^{(2)}$ has a factorization $\overline{\mathrm{g}}^{(2)}=\overline{\mathrm{a}}_{1}^{(2)} \cdot \overline{\mathrm{a}}_{2}^{(2)}$ with respect to $\bar{\alpha}^{(2)}$, where $\overline{\mathrm{a}}_{1}^{(2)}$ corresponds to $\overline{\mathrm{a}}_{1}^{(1)}$ in $\overline{\mathrm{G}}^{(1)}$, which is already known. So we can compute $\overline{\mathrm{a}}_{2}^{(2)}=\left(\overline{\mathrm{a}}_{1}^{(2)}\right)^{-1} \cdot \overline{\mathrm{g}}^{(2)}$.

From the known factorization of $\bar{g}^{(2)}=\overline{\mathrm{a}}_{1}^{(2)} \cdot \overline{\mathrm{a}}_{2}^{(2)}$ with respect to $\bar{\alpha}^{(2)}$ we obtain a factorization of $\overline{\mathrm{g}}^{(3)}=\overline{\mathrm{a}}_{1}^{(3)} \cdot \overline{\mathrm{a}}_{2}^{(3)} \cdot \overline{\mathrm{a}}_{3}^{(3)}$ with respect to $\bar{\alpha}^{(3)}$, where $\overline{\mathrm{a}}_{3}^{(3)}=\left(\overline{\mathrm{a}}_{2}^{(3)}\right)^{-1} \cdot\left(\overline{\mathrm{a}}_{1}^{(3)}\right)^{-1} \cdot \overline{\mathrm{g}}^{(3)}$ and $\overline{\mathrm{a}}_{1}^{(3)}, \overline{\mathrm{a}}_{2}^{(3)}$ are elements in $\overline{\mathrm{G}}^{(3)}$ having their images under the canonical homomorphism as $\overline{\mathrm{a}}_{1}^{(2)}$ and $\overline{\mathrm{a}}_{2}^{(2)}$ in $\overline{\mathrm{G}}^{(2)}$, respectively.

Continuing this process in $(s-1)$ steps we obtain a factorization of $\bar{g}^{(s-1)}=$ $\overline{\mathrm{a}}_{1}^{(s-1)} \ldots \overline{\mathrm{a}}_{\mathrm{s}-1}^{(s-1)}$ with respect to $\bar{\alpha}^{(s-1)}$ in the quotient group $\overline{\mathrm{G}}^{(s-1)}$. Finally we obtain a factorization of $g=a_{1} \cdots a_{s-1} \cdot a_{s}$ with respect to $\alpha$, where $a_{s}=a_{s-1}^{-1} \cdots a_{1}^{-1} \cdot g$, and $a_{1}, \ldots, a_{s-1}$ are the elements in $A_{1}, \ldots, A_{s-1}$ (respectively) giving the corresponding elements $\overline{\mathrm{a}}_{1}^{(s-1)}, \ldots, \overline{\mathrm{a}}_{\mathrm{s}-1}^{(\mathrm{s}-1)}$ in $\overline{\mathrm{G}}^{(s-1)}$.

TheOREM 3.1. Any transversal logarithmic signature for a finite abelian group is tame.

Remark 3.2. Algorithm 2 can be applied to a TLS for a non-abelian group if each subgroup of the chain is normal in the underlying group. In particular, for a Hamiltonian group (a non-abelian group in which any subgroup is normal) any TLS is tame.

\section{Algorithms for factorization with respect to FTLS}

In this section we present algorithms for factoring group elements with respect to a fused transversal logarithmic signature (FTLS) for abelian groups. Let $\alpha=\left[A_{1}, A_{2}, \ldots, A_{s}\right]$ be a transversal logarithmic signature of type $\left(r_{1}, \ldots, r_{s}\right)$ for an abelian group $\mathrm{G}$. We define the following transformations on $\alpha$. 


\section{LOGARITHMIC SIGNATURES FOR ABELIAN GROUPS...}

(i) permute the blocks $A_{i}$ 's,

(ii) permute the elements within blocks $A_{i}$,

(iii) replace a block $A_{i}$ with $A_{i} g$ for some $g \in G$, (as $G$ is abelian, this replacement is in fact an application of a two side transformation on $A_{i}$, namely $h_{i-1}^{-1} A_{i} h_{i}=A_{i} g$, where $\left.g=h_{i-1}^{-1} \cdot h_{i}\right)$,

(iv) replace two blocks $A_{i}$ and $A_{j}$ with a single block $A_{i} \cdot A_{j}=\left\{x y \mid x \in A_{i}\right.$, $\left.y \in A_{j}\right\}$ (we call this operation the fusion of $A_{i}$ and $A_{j}$ ).

A logarithmic signature obtained from a transversal logarithmic signature by applying a finite number of the transformations (i), (ii), (iii) and (iv) is called a fused transversal logarithmic signature (FTLS).

Definition 4.1. A subset $A$ of a finite abelian group $G$ is called periodic if there exists an element $\mathrm{g} \in \mathrm{G} \backslash\{1\}$ with $\mathrm{gA}=\mathrm{A}$. We call such an element $\mathrm{g}$ a period of $A$.

We refer the reader to [12] for details concerning periodicity properties for blocks of logarithmic signatures.

LEMMA 4.2. Let $\beta=\left[\mathrm{B}_{1}, \mathrm{~B}_{2}, \ldots, \mathrm{B}_{\mathrm{t}}\right]$ be a fused transversal logarithmic signature for an abelian group $\mathrm{G}$. Then the following holds:

(i) At least one block $\mathrm{B}_{\mathrm{i}}$ of $\beta$ is periodic.

(ii) Let $x \in \mathrm{B}_{i}$ be a period of $\mathrm{B}_{i}$ and let $\overline{\mathrm{G}}=\mathrm{G} /\langle x\rangle$ be the quotient group of $\mathrm{G}$ modulo the cyclic group $\langle x\rangle$. Then the logarithmic signature $\bar{\beta}=$ $\left[\overline{\mathrm{B}}_{1}, \overline{\mathrm{B}}_{2}, \ldots, \overline{\mathrm{B}}_{\mathrm{t}}\right]$ induced from $\beta$ is a FTLS for $\overline{\mathrm{G}}$.

Proof. (i) Let $\alpha=\left[A_{1}, A_{2}, \ldots, A_{s}\right]$ be a transversal logarithmic signature for $G$, which is used to create $\beta$. Here we may assume that all the blocks of both $\alpha$ and $\beta$ are normalized. Thus the block $A_{1}$, which is a normal subgroup of $G$, is contained in some block $B_{i}$ of $\beta$. It is a simple observation that each element $x \in A_{1} \backslash\{1\}$ is a period of $B_{i}$.

The second statement (ii) is obvious.

Lemma 4.2 can be found in [1]. It is used by Blackburn, $\mathrm{Cid}$ and $\mathrm{Mull}$ a n to prove that FTLS for elementary abelian 2-groups are tame. The authors have given a group argumentation for the proof without showing details. We now show an algorithm for the factorization with respect to an FTLS for any abelian groups based on the Blackburn-Cid-Mullan idea and we determine its complexity.

Again let $\alpha=\left[A_{1}, A_{2}, \ldots, A_{s}\right]$ be a transversal logarithmic signature for an abelian $G$. Let $\beta=\left[B_{1}, B_{2}, \ldots, B_{t}\right]$ be a fused transversal logarithmic signature obtained by applying a finite number of the transformations (i), (ii), (iii) and (iv) to $\alpha$. Let $g$ be an element of $\mathrm{G}$ which we want to factorize by using $\beta$. 


\section{Algorithm 3 Factorization with FTLS}

Input: $G$ : abelian group, $\alpha=\left[A_{1}, A_{2}, \ldots, A_{s}\right]$ a normalized TLS for $G$ constructed from a chain of subgroups $G=G_{1}>G_{2}>\cdots>G_{s+1}=1$ of $G$, $\beta=\left[B_{1}, B_{2}, \ldots, B_{t}\right]$ a FTLS of type $\left(r_{1}, \ldots, r_{t}\right)$ obtained from $\alpha, g \in G$.

Output: $b_{i} \in B_{i}$ such that $g=b_{1} \cdots b_{t}$.

1: (a) Find a period $\chi_{1}$ for a periodic block $B_{i}$.

(b) Consider $\bar{\beta}^{(1)}=\left[\overline{\mathrm{B}}_{1}^{(1)}, \overline{\mathrm{B}}_{2}^{(1)}, \ldots, \overline{\mathrm{B}}_{\mathrm{t}}^{(1)}\right]$ induced by $\beta$ in the quotient group $\overline{\mathrm{G}}^{(1)}=\mathrm{G} /\left\langle x_{1}\right\rangle$. (Then $\bar{\beta}$ is an FTLS for $\overline{\mathrm{G}}$ by Lemma 4.2. $\bar{\beta}$ is of type $\left(r_{1}, \ldots, r_{i-1}, r_{i} / \delta_{1}, r_{i+1}, \ldots, r_{t}\right)$, where $\delta_{1}$ is the order of $x_{1}$.)

(c) Define $\bar{g}^{(1)}$ to be the induced element of $g$ in the quotient group $\bar{G}^{(1)}$. Repeat (a), (b) and (c) for $\bar{\beta}^{(1)}, \bar{G}^{(1)}$ and $\bar{g}^{(1)}$ to obtain $\bar{\beta}^{(2)}, \bar{G}^{(2)}$ and $\bar{g}^{(2)}$, where $\overline{\mathrm{G}}^{(2)}=\overline{\mathrm{G}}^{(1)} /\left\langle\bar{\chi}_{2}\right\rangle$ and $\bar{\chi}_{2}$ is a period of some block $\overline{\mathrm{B}}_{j}^{(1)}$. Continuing this process we eventually obtain a trivial $\operatorname{LS} \bar{\beta}^{(u)}$ for the trivial group $\bar{G}^{(u)}$ after a finite number of steps, say $u$. Also, the induced element $\bar{g}^{(\mathfrak{u})} \in \overline{\mathbf{G}}^{(\mathfrak{u})}$ becomes the identity element.

2: Working backward from $\bar{\beta}^{(\mathfrak{u})}, \bar{\beta}^{(\mathfrak{u}-1)}, \ldots$ to $\bar{\beta}^{(1)}$ we can factorize $\mathrm{g}$ with respect to $\beta$ as follows. Here, we describe one step of the factorization process. First note that $\bar{\beta}^{(i)}$ and $\bar{\beta}^{(i-1)}$ have all blocks of the same type except one block of $\bar{\beta}^{(i-1)}$ containing the period $\bar{\chi}^{(i-1)}$ which is used to define $\bar{\beta}^{(i)}$ from $\bar{\beta}^{(i-1)}$. W.l.o.g. we may assume that this periodic block is the first block $\overline{\mathrm{B}}_{1}^{(\mathrm{i}-1)}$ of $\bar{\beta}^{(i-1)}=\left[\overline{\mathrm{B}}_{1}^{(\mathrm{i}-1)}, \overline{\mathrm{B}}_{2}^{(\mathrm{i}-1)}, \ldots, \overline{\mathrm{B}}_{\mathrm{t}}^{(\mathrm{i}-1)}\right]$. Let $\bar{\beta}^{(i)}=$ $\left[\overline{\mathrm{B}}_{1}^{(\mathrm{i})}, \overline{\mathrm{B}}_{2}^{(\mathrm{i})}, \ldots, \overline{\mathrm{B}}_{\mathrm{t}}^{(\mathrm{i})}\right]$. Assume by induction that $\overline{\mathrm{g}}^{(\mathrm{i})}=\overline{\mathrm{b}}_{1 j_{1}}^{(\mathrm{i})} \cdot \overline{\mathrm{b}}_{2 \mathrm{j}_{2}}^{(\mathrm{i})} \cdots \overline{\mathrm{b}}_{\mathrm{t} \mathrm{j}_{\mathrm{t}}}^{(\mathrm{i})}$ is a known factorization of $\bar{g}^{(i)}$ with respect to $\bar{\beta}^{(i)}$ (i.e., $\bar{b}_{j}^{(i)} \in \bar{B}_{j}^{(i)}, j=$ $1, \ldots, t)$. Now $\bar{g}^{(i-1)}$ is known since $\bar{g}^{(i)}$ is known by the induction assumption. Let $\bar{g}^{(i-1)}=\bar{b}_{1 k_{1}}^{(i-1)} \cdot \bar{b}_{2 k_{2}}^{(i-1)} \cdots \bar{b}_{t k_{t}}^{(i-1)}$ be a factorization of $\bar{g}^{(i-1)}$ with respect to $\bar{\beta}^{(i-1)}$. Then we have $k_{m}=j_{m}$ for $m=2, \ldots$, t.

Hence the element $\bar{b}_{1 k_{1}}^{(i-1)} \in \bar{B}_{1}^{(i-1)}$ is uniquely determined by

$$
\overline{\mathrm{b}}_{1 \mathrm{k}_{1}}^{(\mathrm{i}-1)}=\overline{\mathrm{g}}^{(\mathrm{i}-1)} \cdot\left(\overline{\mathrm{b}}_{\mathrm{t} \mathrm{j}_{\mathrm{t}}}^{(\mathrm{i}-1)}\right)^{-1} \cdots\left(\overline{\mathrm{b}}_{2 \mathrm{j}_{2}}^{(\mathrm{i}-1)}\right)^{-1} .
$$

Here we assume that all the blocks $B_{i}$ 's of $\beta$ are normalized. The main idea of factoring with respect to an FTLS for elementary abelian 2-groups, see 1, is as follows: Find a period $x$ for a certain block of $\beta$ and transform $\beta$ to $\bar{\beta}$ in the quotient group $\bar{G}=\mathrm{G} /\langle x\rangle$. Again $\bar{\beta}$ is an FTLS for $\overline{\mathrm{G}}$ by Lemma 4.2 , so the process is repeated with $\bar{\beta}$ and $\bar{G}$ until we reach the trivial quotient group, and the resulting FTLS becomes a trivial logarithmic signature. In this process we also keep track of the induced elements of $\mathrm{g}$ in the quotient groups. 


\section{LOGARITHMIC SIGNATURES FOR ABELIAN GROUPS...}

Based on the idea of B la c k b u r n, C id and M u lla n we show the factoring algorithm with respect to an FTLS for abelian groups.

In the following we attempt to determine the complexity of Algorithm 3 for elementary abelian p-groups. For the sake of simplicity we also asumme that $r_{i}=r$ for $i=1, \ldots, t$ and $\left|A_{i}\right|=z$ for $i=1, \ldots, s$. If we would not have the assumption, it would be more involved to compute the complexity.

Let $G$ be an elementary abelian p-group. Let $\alpha=\left[A_{1}, A_{2}, \ldots, A_{s}\right]$ be a TLS constructed from a chain of subgroups $\mathrm{G}=\mathrm{G}_{1}>\mathrm{G}_{2}>\cdots>\mathrm{G}_{\mathrm{s}+1}=1$ of $\mathrm{G}$ of type $(z, \ldots, z)$ (i.e., $\left|A_{i}\right|=z$ for all $\left.i=1, \ldots, t\right)$. We also assume that $r_{i}=r$ for all $i=1, \ldots, t$. So, we have $r_{i}=p^{e}$ for $i=1, \ldots, t$.

One main part of the complexity of the algorithm is the finding of periodic elements in the process of constructing induced FTLS for the quotient group $\overline{\mathrm{G}}^{(j)}$ for each $j=1, \ldots, \mathfrak{u}$, where $u$ is the smallest number such that the quotient group $\overline{\mathrm{G}}^{(\mathrm{u})}$ becomes the identity group.

To start with we have to find a period in a certain block of $\beta$. There are $t$ possible choices for such a block, say $B_{i}$. For an $x \in B_{i}$, verifying whether $x$ is a period, i.e., $x B_{i}=B_{i}$, requires a complexity of $\Theta\left(\left|B_{i}\right| \log _{2}\left|B_{i}\right|\right)$. This complexity is composed of computing $\left|B_{i}\right|$ times multiplications $x \cdot b_{i 1}, \ldots, x \cdot b_{i r}$ and of checking if $x \cdot b_{i j} \in B_{i}$. The checking has a complexity $\Theta\left(\log _{2}\left|B_{i}\right|\right)$, if block $B_{i}$ is sorted (otherwise it would be of complexity $\Theta\left(\left|B_{i}\right|\right)$ ). Therefore, we will assume that each block $B_{i}$ is sorted once. Sorting of $B_{i}$ has a complexity of $\Theta\left(\left|B_{i}\right| \log _{2}\left|B_{i}\right|\right)$. For each step of moving to the quotient group the unique block of $\bar{\beta}^{(k)}$ whose size is decreased needs also to be sorted (more precisely, if $x$ is a period in $\bar{B}_{i}^{(k-1)}$, the block $\bar{B}_{i}^{(k)}$ of $\bar{\beta}^{(k)}$ in the quotient group $\bar{G}^{(k)}=\bar{G}^{(k-1)} /\langle x\rangle$ is of size $\left|\bar{B}_{i}^{(k)}\right|=\left|\bar{B}_{i}^{(k-1)}\right| / p$ and we have to sort $\left.\bar{B}_{i}^{(k)}\right)$.

As the computation of pointer elements $b_{i}$ 's in the factorization of $g$ in step 2 is deterministic, we may regard the time spent for this step as being constant and therefore its complexity will be neglected.

The total number of operations in step 1 comprises the number of operations for finding periods, denoted by $\mathrm{A}$, and the number of operations for block sorting, denoted by B. Here we have

$$
\begin{aligned}
A & =t\left(\left(r / p^{0}\right)^{2} \log _{2}\left(r / p^{0}\right)+(r / p)^{2} \log _{2}(r / p)+\cdots+\left(r / p^{e-1}\right)^{2} \log _{2}\left(r / p^{e-1}\right)\right) \\
& =\frac{t}{\log _{p} 2} \sum_{i=1}^{e}\left(p^{2}\right)^{i} i
\end{aligned}
$$

and

$$
\begin{aligned}
B & =t\left(\left(r / p^{0}\right) \log _{2}\left(r / p^{0}\right)+(r / p) \log _{2}(r / p)+\cdots+\left(r / p^{e-1}\right) \log _{2}\left(r / p^{e-1}\right)\right) \\
& =\frac{t}{\log _{p} 2} \sum_{i=1}^{e} p^{i} i .
\end{aligned}
$$




$$
\text { PAVOL SVABA - TRAN VAN TRUNG - PAUL WOLF }
$$

By using the formula

$$
\sum_{i=1}^{n} i x^{i}=\frac{n x^{n+2}-(n+1) x^{n+1}+x}{(x-1)^{2}},
$$

where $x \neq 1$, the total number of operations in step 1 amounts to

$$
\begin{aligned}
A+B= & \frac{t}{\log _{p} 2}\left(\frac{e\left(p^{2}\right)^{e+2}-(e+1)\left(p^{2}\right)^{e+1}+p^{2}}{\left(p^{2}-1\right)^{2}}\right. \\
& \left.+\frac{e p^{e+2}-(e+1) p^{e+1}+p}{(p-1)^{2}}\right) \\
= & \Theta\left(\frac{t}{\log _{p} 2}\left(p^{2}\right)^{e} \log _{p} p^{e}\right) \\
= & \Theta\left(\operatorname{tr}^{2} \log _{2} r\right) .
\end{aligned}
$$

We record the result of the above analysis in the following theorem.

TheOrem 4.3. Let $\mathrm{G}$ be a finite abelian p-group and let $\beta$ be an FTLS of type $\left(\mathrm{r}_{1}, \mathrm{r}_{2}, \ldots, \mathrm{r}_{\mathrm{t}}\right)=(\mathrm{r}, \mathrm{r}, \ldots, \mathrm{r})$ for $\mathrm{G}$ obtained from a TLS of type $\left(z_{1}, \ldots, z_{\mathrm{s}}\right)=$ $(z, z, \ldots, z)$. Then the factorization of an element $\mathrm{g} \in \mathrm{G}$ with respect to $\beta$ using Algorithm 3 has a complexity of $\Theta\left(\operatorname{tr}^{2} \log _{2} r\right)$.

The complexity as given in Theorem 4.3 shows that if the sizes for $r$ are small, Algorithm 3 could still be considered as "efficient", but if $r$ is getting large, Algorithm 3 will no longer be efficient. And because of the term $r^{2}$ involved in the complexity estimate, Algorithm 3 cannot be used to prove the tameness of FTLS for abelian groups (i.e., the complexity $\Theta\left(\operatorname{tr}^{2} \log _{2}(r)\right)$ cannot be expressed in terms of $\mathrm{O}\left(\boldsymbol{w}^{\mathrm{c}}\right)$, where $\mathrm{c}$ is a constant and $\mathrm{w}$ is the width of $\left.\mathrm{G}\right)$.

In the next section we show that if the information of the transformations used for generating an FTLS $\beta$ from a TLS is known, then we can construct a factoring algorithm proving the tameness of $\beta$.

\subsection{Factorization with respect to FTLS by using trapdoor information}

Assume that an FTLS $\beta$ for an abelian group $\mathcal{G}$ is constructed from a TLS $\alpha$ using the four transformations (i), (ii), (iii) and (iv) as described at the beginning of the section. To be more precise, let the TLS $\alpha=\left[A_{1}, A_{2}, \ldots, A_{s}\right]$ of type $\left(z_{1}, \ldots, z_{\mathrm{s}}\right)$ be derived from a chain of subgroups $\mathrm{G}=\mathrm{G}_{0}>\mathrm{G}_{1}>\cdots>\mathrm{G}_{\mathrm{s}}=1$ of $\mathrm{G}$.

In general, there is no particular order of using the transformations (i), (ii), (iii) and (iv), but for the sake of clarity we will generate an FTLS according to the following steps. 


\section{LOGARITHMIC SIGNATURES FOR ABELIAN GROUPS...}

(T1) (Fusion) Perform a fusion of the blocks of $\alpha$. The fusion transformation (iv) will be done as follows.

- Select a permutation $\varphi \in S_{\mathrm{s}}$ and compute a logarithmic signature $\alpha^{\prime}$ from $\alpha$ by

$$
\alpha^{\prime}=\left[A_{1}^{\prime}, \ldots, A_{s}^{\prime}\right]=\left[A_{\varphi(1)}, \ldots, A_{\varphi(s)}\right] .
$$

- Select a partition $\mathrm{P}=\left\{\mathrm{P}_{1}, \ldots, \mathrm{P}_{\mathrm{t}}\right\}$ on the set $\{1, \ldots, s\}$ with $\mathrm{P}_{1}=$ $\left\{1, \ldots, i_{1}\right\}, P_{2}=\left\{i_{1}+1, \ldots, i_{2}\right\}, \ldots, P_{t}=\left\{i_{s-1}+1, \ldots, i_{s}\right\}$ with $\left|P_{j}\right|=u_{j}$, for $j=1, \ldots, t$. Fusing the blocks of $\alpha^{\prime}$ according to this partition yields a logarithmic signature $\beta^{\prime}:=\left[B_{1}^{\prime}, \ldots, B_{t}^{\prime}\right]$ of type $\left(r_{1}, \ldots, r_{t}\right)$ with

$$
B_{j}^{\prime}=A_{i_{j-1}+1}^{\prime} \cdot A_{i_{j-1}+2}^{\prime} \cdots A_{i_{j}}^{\prime},
$$

and $r_{j}=\left|A_{i_{j-1}+1}^{\prime}\right| \cdot\left|A_{i_{j-1}+2}^{\prime}\right| \cdots\left|A_{i_{j}}^{\prime}\right|$ for $j=1, \ldots, t$ and $i_{0}=0$.

(i.e., each block $B_{i}^{\prime}$ is obtained by fusing certain consecutive blocks of $\alpha^{\prime}$.)

(T2) Select random permutations $\pi_{j} \in S_{r_{j}}, j=1, \ldots, t$. Permute the positions of the elements of each block $B_{j}^{\prime}$ with permutation $\pi_{j}$. Let $\beta^{\prime \prime}=\left[B_{1}^{\prime \prime}, \ldots, B_{t}^{\prime \prime}\right]$ denote the resulting logarithmic signature obtained from $\beta^{\prime}$ after this step.

(T3) Select random elements $g_{j} \in G$ and replace each block $B_{j}^{\prime \prime}$ of $\beta^{\prime \prime}$ with $B^{\prime \prime \prime}:=B_{j}^{\prime \prime} \cdot g_{j}$. The resulting object is a logarithmic signature $\beta^{\prime \prime \prime}=$ $\left[\mathrm{B}_{1}^{\prime \prime \prime}, \ldots, \mathrm{B}_{\mathrm{t}}^{\prime \prime \prime}\right]$.

(T4) Select a random permutation $\xi \in S_{t}$ and permute the blocks of $\beta^{\prime \prime \prime}$ by using $\xi$. The result obtained from this last step is our constructed FTLS $\beta=\left[B_{1}, \ldots, B_{t}\right]$.

We call the information about the transformations T1, T2, T3 and T4, which are used to generate an FTLS $\beta$ from a TLS $\alpha$, the trapdoor information.

Proposition 4.4. Let $\alpha:=\left[\mathrm{A}_{1}, \ldots, \mathrm{A}_{\mathrm{s}}\right]$ be a transversal logarithmic signature for an abelian group $\mathrm{G}$. Let $\beta^{\prime}:=\left[\mathrm{B}_{1}^{\prime}, \ldots, \mathrm{B}_{\mathrm{t}}^{\prime}\right]$ be a fused transversal logarithmic signature for $\mathrm{G}$ obtained from $\alpha$ by using (only) the fusion transformation $\mathrm{T} 1$. Then $\beta^{\prime}$ is equivalent to a logarithmic signature $\alpha^{\prime}$ obtained from $\alpha$ by permuting its blocks with the permutation used by $\mathrm{T} 1$.

P r o of. Now suppose that $\beta^{\prime}$ is given. Let $\alpha^{\prime}=\left[A_{1}^{\prime}, \ldots, A_{s}^{\prime}\right]$ be the logarithmic signature obtained from $\alpha$ by using the permutation $\varphi \in S_{\mathrm{S}}$ for transformation T1, i.e.,

$$
\alpha^{\prime}=\left[A_{1}^{\prime}, \ldots, A_{s}^{\prime}\right]=\left[A_{\varphi(1)}, \ldots, A_{\varphi(s)}\right] .
$$

Then it is clear that $\beta^{\prime}$ is equivalent to $\alpha^{\prime}$.

As a consequence of Proposition 4.4 we see that instead of factoring with respect to an FTLS $\beta$ we can factorize with respect to $\alpha$ by using the knowledge of transformations T1, T2, T3 and T4. This is presented in the following algorithm. 


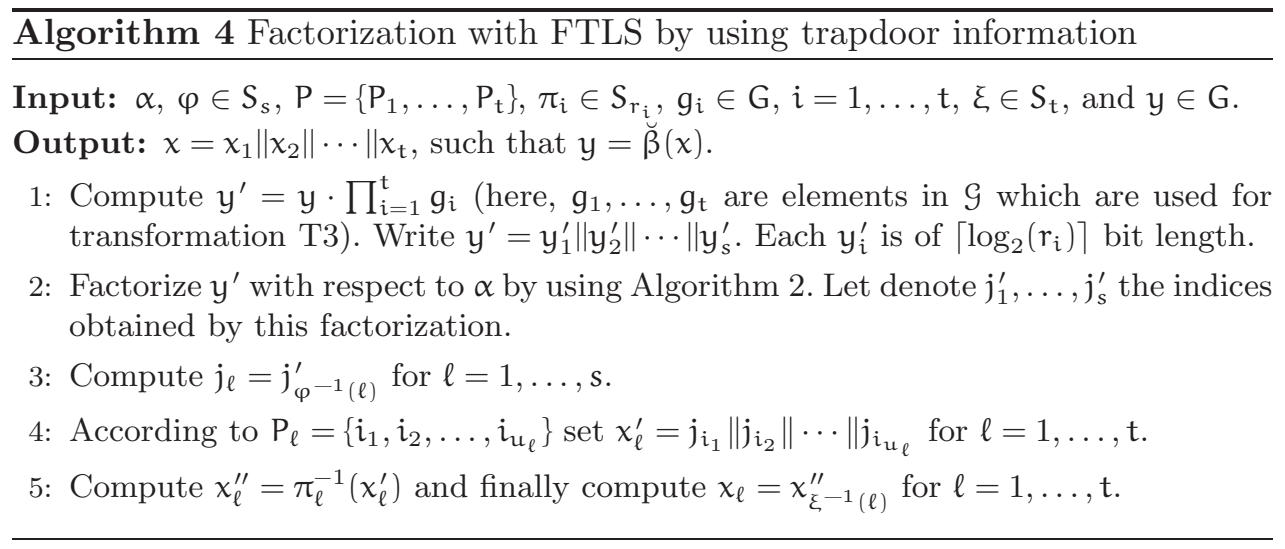

In Algorithm 4 we may assume that the performing steps 1, 3, 4, 5 will take a constant time. Thus the complexity for factoring $y$ with respect to $\beta$ is reduced to the complexity of factoring $y^{\prime}$ with respect to the TLS $\alpha$ in the step 2, which is $\mathrm{O}(w)$ by Theorem 3.1. where $w=\left\lceil\log _{2}|\mathrm{G}|\right\rceil$. Thus we have the following theorem.

Theorem 4.5. Let $\beta:=\left[\mathrm{B}_{1}, \ldots, \mathrm{B}_{\mathrm{t}}\right]$ be an FTLS constructed from a TLS $\alpha:=\left[A_{1}, \ldots, A_{s}\right]$ for an abelian group $G$ by using the transformations $\mathrm{T} 1, \mathrm{~T} 2$, T3 and $\mathrm{T} 4$. Then $\beta$ is tame if the trapdoor information about these transformations is known.

\section{Conclusion}

We have presented factorization algorithms and their computational complexities for the classes of transversal and fused transversal logarithmic signatures for finite abelian groups. The results have shown that transversal logarithmic signatures are tame, however, fused transversal logarithmic signatures are tame when trapdoor information is used. We have also presented a factorization algorithm for fused transversal logarithmic signatures based on the idea of Blackburn, Cid and Mullan and computed its complexity. It is an interesting open problem to decide whether or not fused transversal logarithmic signatures for abelian groups are tame without using the trapdoor information.

\section{REFERENCES}

[1] BLACKBURN, S. R.-CID, C.-MULLAN, C.: Cryptanalysis of the MST 3 public key cryptosystem, J. Math. Crypt. 3 (2009), 321-338.

[2] LEMPKEN, W.-MAGLIVERAS, S. S.-TRAN VAN TRUNG-WEI, W.: A public key cryptosystem based on non-abelian finite groups, J. Cryptology 22 (2009), 62-74. 


\title{
LOGARITHMIC SIGNATURES FOR ABELIAN GROUPS...
}

[3] MAGLiVERAS, S. S.-OBERG, B. A.-SURKAN, A. J.: A new random number generator from permutation groups, Rend. Sem. Mat. Fis. Milano 54 (1984), 203-223.

[4] MAGLIVERAS, S. S.: A cryptosystem from logarithmic signatures of finite groups, in: Proc. of the 29th Midwest Symposium on Circuits and Systems (M. Ismail, ed.), Lincoln, NE, 1986, Elsevier Publ. Comp., 1986, pp. 972-975.

[5] MAGLIVERAS, S. S.-MEMON, N. D.: Random permutations from logarithmic signatures, in: Computing in the 90's, 1st Great Lakes Comp. Sci. Conf. (N. A. Sherwani et al., eds.), Kalamazoo, USA, 1989, Lecture Notes in Comput. Sci., Vol. 507 Springer-Verlag, Berlin, 1989, pp. 91-97.

[6] MAGLIVERAS, S. S.-MEMON, N. D.: The algebraic properties of cryptosystem PGM, J. Cryptology 5 (1992), 167-183.

[7] MAGLIVERAS, S. S.-STINSON, D. R.-TRAN VAN TRUNG: New approaches to designing public key cryptosystems using one-way functions and trapdoors in finite groups, J. Cryptology 15 (2002), 285-297.

[8] MAGLIVERAS, S. S.-SVABA, P.-TRAN VAN TRUNG-ZAJAC, P.: On the security of a realization of cryptosystem $\mathrm{MST}_{3}$, Tatra Mt. Math. Publ. 41 (2008), 1-13.

[9] MARQUARDT, P.-SVABA, P.-TRAN VAN TRUNG: Pseudorandom number generators based on random covers for finite groups, Des. Codes Cryptogr. 64 (2012), 209-220.

[10] SVABA, P.-TRAN VAN TRUNG: On generation of random covers for finite groups, Tatra Mt. Math. Publ. 37 (2007), 105-112.

[11] SVABA, P.-TRAN VAN TRUNG: Public key cryptosystem MST3: cryptanalysis and realization, J. Math. Crypto. 4 (2010), 271-315.

[12] SZABÓ, S.: Topics in Factorization of Abelian Groups. Birkhäuser Verlag, Berlin, 2004.

[13] VASCO, M.I. G.-DEL POZO, AIP.-DUARTE, P.T.: A note on the security of $\mathrm{MST}_{3}$ Des. Codes Cryptogr. 55 (2010), 189-200.

Received July 31, 2013

\author{
Pavol Svaba \\ Institute of Business Inform. Technology \\ Lucerne University of Appl. Sci. and Arts \\ Zentralstrasse 9 \\ CH-6002 Lucerne \\ SWITZERLAND \\ E-mail: pavol.svaba@hslu.ch \\ Tran van Trung \\ Institute for Experimental Math. \\ University of Duisburg-Essen \\ Ellernstrasse 29 \\ D-45326 Essen \\ GERMANY \\ E-mail: trung@iem.uni-due.de \\ Paul Wolf \\ University of Paderborn \\ Warburger Strasse 100 \\ D-33098 Paderborn \\ GERMANY \\ E-mail: paul.wolf@uni-paderborn.de
}

